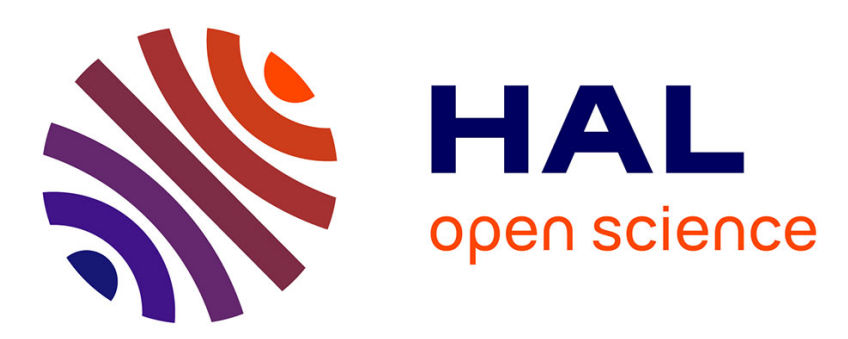

\title{
Remarks on stochastic properties of materials through finite deformations
}

\author{
Roger Ghanem, Christian Soize
}

\section{To cite this version:}

Roger Ghanem, Christian Soize. Remarks on stochastic properties of materials through finite deformations. International Journal for Multiscale Computational Engineering, 2015, 13 (4), pp.367-374. 10.1615/IntJMultCompEng.2015013959 hal-01162152

\section{HAL Id: hal-01162152 \\ https://hal.science/hal-01162152}

Submitted on 31 Aug 2015

HAL is a multi-disciplinary open access archive for the deposit and dissemination of scientific research documents, whether they are published or not. The documents may come from teaching and research institutions in France or abroad, or from public or private research centers.
L'archive ouverte pluridisciplinaire HAL, est destinée au dépôt et à la diffusion de documents scientifiques de niveau recherche, publiés ou non, émanant des établissements d'enseignement et de recherche français ou étrangers, des laboratoires publics ou privés. 


\title{
Remarks on Stochastic Properties of Materials Through Finite Deformations ${ }^{\text {tर }}$
}

\author{
R. Ghanem*,a C. Soize ${ }^{\mathrm{b}}$ \\ ${ }^{a}$ University of Southern California, 210 KAP Hall, Los Angeles, CA 90089, United States \\ ${ }^{b}$ Université Paris-Est, Laboratoire Modélisation et Simulation Multi-Echelle, MSME UMR 8208 \\ CNRS, 5 bd Descartes, 77454 Marne-la-Vallée Cedex 2, France
}

\begin{abstract}
Some remarks are provided concerning the effects of geometrical transformations, such as those encountered in a manufacturing process, on the stochastic character of corresponding material properties. It is shown that, in general, even secondorder statistical properties are transformed in a non-trivial manner as the material undergoes arbitrary transformation.
\end{abstract}

Key words: Heterogeneous material, finite deformations, stochastic material properties, random media, random elasticity field, multiscale.

\section{Introduction}

Recent developments and progress in computational stochastic mechanics require consistent probabilistic models of material properties. A number of difficulties appear in initial efforts at integrating stochastic models with computational mechanics tools due to both lack of experimental measurements as well as lack of methods for constructing physically consistent stochastic models for such materials.

It is well known that finite deformations of a material, such as those occurring during the manufacturing process, induce modifications of the material proper-

\footnotetext{
International Journal for Multiscale Computational Engineering, 2015, doi: 10.1615/IntJMultCompEng.2015013959.

* Corresponding author

Email addresses: ghanem@usc.edu (R. Ghanem), christian.soizeduniv-paris-est.fr(C. Soize)
}

Preprint submitted to International Journal for Multiscale Computational Engineering 
ties which are generally highly complex and which cannot, a priori, be summarized in just a geometrical transformation. This paper presents a framework for investigating the manner in which stochastic properties of heterogeneous materials are transformed under finite deformations, in considering not only the geometrical aspects but also the physical modifications of the material induced by the finite deformation. More precisely, the analysis is focused on heterogeneous materials that are assumed to be linear elastic in the undeformed configuration, and which are transformed by a finite deformation into a heterogeneous material for which the linear elastic properties are studied in the deformed configuration. The statistical fluctuations in the properties of materials, whether in the deformed or undeformed configurations, are induced by microstructure heterogeneity. The proposed framework does not address situations involving phase transformation during the processing stage. Thus for instance, while deformation of the fiber reinforcement matrix during the manufacturing of composites is covered, the solidification and curing of resin during that same process would require additional analysis not covered in this paper. That analysis, however, would have to be carried out using the consistent statistical descriptions of the fiber reinforcement as described in this paper. Another example covered in the present paper involves transformations induced by cold rolling and forming of metallic sheets. The results of this paper demonstrate that the properties of the statistical fluctuations of material properties in the deformed configuration, including their probability density functions, correlation structure and stationarity, are functions of the manner in which the deformation affects both the microstructure and the geometry. Developing multiscale statistical models for material properties must therefore take into account the details of any large deformation to which the material has been subjected. These properties will generally be different from the statistical properties of the material in the undeformed stage.

The paper demonstrates that even with an elementary analysis of the material deformation process, some important properties of the statistical fluctuations can be inferred. The paper stops short, however, of prescribing a procedure for synthesizing the stochastic processes in the deformed stage.

\section{Definition of the Problem}

It is assumed that the data associated with the problem consist of a natural configuration of a heterogeneous linear elastic material occupying domain $\Omega_{0}$ and a manufacturing process, defined by a deterministic mapping $\mathbf{f}$, which transforms $\Omega_{0}$ into a deformed configuration $\Omega$. It is further assumed that the statistical prop- 
erties of the parameters of the constitutive equation of the material at $\Omega_{0}$ are known and can generally be deduced from an experimental identification. This statistical behavior is typically induced by the interaction of the scale at which the constitutive equation is described with subscales at which significant statistical variability prevents a deterministic analysis. This behavior is prevalent in materials with random microstructure $[8,9,15]$. The objective of the present analysis is then to construct the statistical properties of the parameters of the constitutive equation in the domain of linear elasticity of the heterogeneous material at the new deformed configuration $\Omega$. Figure (1) depicts the basic elements of this problem. The new configuration is then used as the new reference configuration for further engineering analysis. It is indeed this new configuration that is often of interest as it represents manufactured components to be used in design.

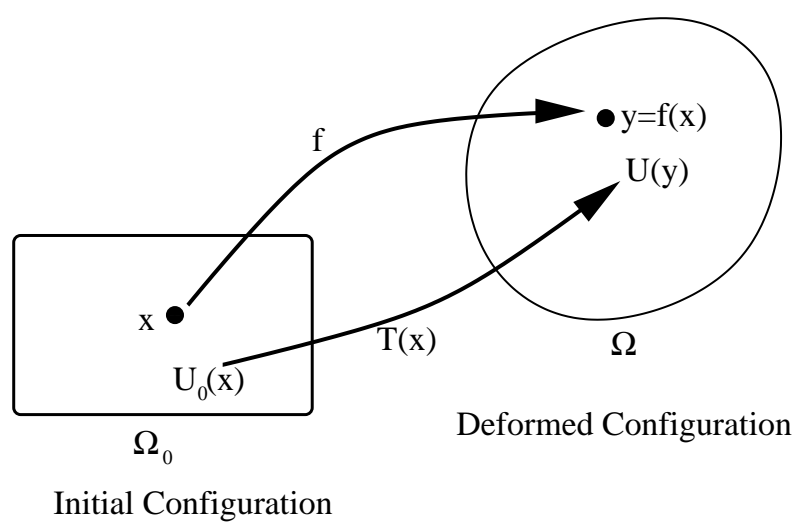

Figure 1: Transformation from $\Omega_{0}$ to $\Omega$.

This problem is ubiquitous in the analysis of systems with stochastic materials for which the statistical properties are required. For instance, stochastic finite element analyses applied to manufactured components require knowledge of the statistical properties of the material in the geometry corresponding to its manufactured configuration $\Omega$. These statistical properties are usually different from the properties in $\Omega_{0}$.

Two cases will be shown to be relevant in this context. The first case corresponds to the usual situation in deterministic continuum mechanics for which the material properties in configuration $\Omega_{0}$ are transported to deformed configuration $\Omega$. Such a transport is defined by a deterministic linear pointwise operator depending on each point $\mathbf{x}$ in $\Omega_{0}$. This case corresponds to the situation for which the statistical 
properties of the random microstructure is not modified by mapping $\mathbf{f}$ defining the manufacturing process. This does not mean that the statistical properties of the material are the same for configurations $\Omega_{0}$ and $\Omega$, in view of the material transport associated with $\mathbf{f}$. The second case corresponds to the most general situations where mapping $\mathbf{f}$ induces such changes in the random microstructure that the statistics of the material parameters are modified as domain $\Omega_{0}$ is transformed into domain $\Omega$. In this case, the material transformation is clearly a random operator which depends both on $\mathbf{x}$ and the random microstructure.

A choice has to be made at this point regarding the statistical modeling of the random properties of the material in the natural configuration defined by $\Omega_{0}$. Clearly, associating the randomness to the presence of a random microstructure leads the parameters of the constitutive equation to be modeled as stochastic fields. From the theory of probability, a stochastic field is completely described by its system of joint distributions. Obviously, this system cannot be directly identified from experimental data and an alternative characterization must be introduced. It should however be noted that the response of systems for which the reference configuration is the manufactured configuration $\Omega$, is highly nonlinear with respect to the material parameters such as Young modulus and Poisson coefficient for an isotropic elastic medium. Consequently a second-order description of these stochastic fields is not sufficient. Representations that provide an equivalent level of information to that available through the system of joint distributions is required. Such a representation is provided, for instance, through the polynomial chaos decomposition. This representation will be used in the sequel.

\section{Mathematical Characterization of the Transformations}

Two transformations are significant to the present analysis. The first one deals with the nonlinear mapping $\mathbf{f}$ which transforms the material domain from its $\Omega_{0}$ to its $\Omega$ configuration. The second transformation deals with the linear pointwise operator which carries the material properties associated with $\Omega_{0}$ to those associated with $\Omega$. We assume that the first mapping $f$ is deterministic, while the second mapping is potentially uncertain. This assumption assumes that while the manufacturing process is controllable at the coarse scale, the modifications it imparts on the substructure are not always predictable. This section defines the material properties in $\Omega$ and $\Omega_{0}$ and highlights their characteristics. Following that, the stochastic properties of the transformed material quantities are also analyzed. 


\subsection{Defining the Nonlinear Mapping $f$}

As defined above, let $\Omega_{0}$ be a bounded domain of $\mathbb{R}^{n}$ occupied by the material and referring to its natural configuration. Domain $\Omega_{0}$ is equipped with the measure $d \mu_{0}(\mathbf{x})$. For instance if $\Omega_{0}$ is a volume in $\mathbb{R}^{3}, d \mu_{0}(\mathbf{x})$ is the Lebesgue measure in $\mathbb{R}^{3}$; if $\Omega_{0}$ is a surface in $\mathbb{R}^{3}, d \mu_{0}(\mathbf{x})$ is the corresponding surface measure. Let $\mathbf{f}$ be the mapping introduced above. To simplify the presentation, it is assumed that this mapping $\mathbf{f}$ is one-to-one from $\Omega_{0}$ onto a bounded domain, $\Omega$, of $\mathbb{R}^{n}$,

$$
\begin{aligned}
& \Omega_{0} \stackrel{\mathbf{f}}{\longrightarrow} \\
& \mathbf{x} \stackrel{\mathbf{f}}{\mapsto} \\
& \mathbf{y}=\mathbf{f}(\mathbf{x})
\end{aligned}
$$

Consequently, $\mathbf{f}^{-1}$ exists from $\Omega$ onto $\Omega_{0}$. We assume furthermore that $\mathbf{f}$ and $\mathbf{f}^{-1}$ are differentiable. These assumptions can be readily relaxed by defining this mapping in a piecewise manner over adjacent regions in $\Omega_{0}$ on which it is bijective; this is essentially equivalent to using manifold theory. The measure $d \mu_{0}(\mathbf{x})$ on $\Omega_{0}$ is transported by $\mathbf{f}$ into a measure $d \mu(\mathbf{y})$ on $\Omega$ such that $d \mu(\mathbf{y})=d \mu_{0}(\mathbf{x})$. Using equation (1) yields,

$$
d \mu(\mathbf{y})=|\operatorname{det}[J(\mathbf{y})]| d \mu_{0}\left(\mathbf{f}^{-1}(\mathbf{y})\right),
$$

in which $\operatorname{det}[J(\mathbf{y})]$ is the determinant of the jacobian matrix $[J(\mathbf{y})]$ of transformation $\mathbf{f}^{-1}$. This jacobian matrix, assumed to be invertible, is given by,

$$
[J(\mathbf{y})]=\nabla_{\mathbf{y}}\left(\mathbf{f}^{-1}(\mathbf{y})\right)
$$

where $\nabla_{\mathbf{y}}$ is the gradient with respect to $\mathbf{y}$.

\subsection{Transforming the Material Properties}

Consider an $\mathbb{R}^{\nu}$-valued vector $\mathbf{U}_{0}(\mathbf{x})$ in the natural configuration $\Omega_{0}$ associated with a tensorial quantity defined in a local coordinate system $\mathcal{E}_{0}(\mathbf{x})$ depending on point $\mathbf{x}$. The corresponding $\mathbb{R}^{\nu}$-valued vector $\mathbf{U}(\mathbf{y})$ in deformed configuration $\Omega$ is defined with respect to another coordinate system $\mathcal{E}(\mathbf{y})$ which depends on $\mathbf{y} \in \Omega$. A linear mapping can thus be defined from $\mathbb{R}^{\nu}$ into $\mathbb{R}^{\nu}$ which maps $\mathbf{U}_{0}(\mathbf{x})$ into $\mathbf{U}(\mathbf{y})$. This linear mapping is denoted by the deterministic or random $\nu \times \nu$ real matrix $[T(\mathbf{x})]$ such that,

$$
\mathbf{U}(\mathbf{y})=[T(\mathbf{x})] \mathbf{U}_{0}(\mathbf{x}), \quad \mathbf{y}=\mathbf{f}(\mathbf{x})
$$


For instance, vector $\mathbf{U}_{0}(\mathbf{x})$ could represent the vector consisting of the 21 constants in the elasticity tensor $\mathcal{C}_{0}(\mathbf{x})$, represented in coordinate system $\mathcal{E}_{0}(\mathbf{x})$ attached to $\Omega_{0}$, and associated with a general nonhomogeneous anisotropic material. Moreover, let $\mathcal{C}(\mathbf{y})$ denote the elasticity tensor corresponding to $\mathcal{C}_{0}(\mathbf{x})$, in the deformed configuration $\Omega$. Tensor $\mathcal{C}(\mathbf{y})$ is defined relative to a coordinate system $\mathcal{E}(\mathbf{y})$ attached to $\Omega$. The $\mathbb{R}^{\nu}$-valued vector $\mathbf{U}(\mathbf{y})$ consists then of the 21 constants in tensor $\mathcal{C}(\mathbf{y})$.

The two cases introduced in Section 4 correspond, respectively, to the cases where matrix $[T(\mathbf{x})]$ is deterministic and random.

\section{Stochastic Properties of Transformed Material Properties}

As explained above, the randomness in the transformed material properties must be derived from either random properties in the natural configuration, or a random evolution of the materials between the two configurations. The statistical characterization of the material properties in the natural configuration, described in the next subsection, is thus paramount for the characterization of these properties in the deformed configuration.

\subsection{Characterization of Stochastic Properties in Natural Configuration}

If the material properties in the natural configuration, represented by $\mathbf{U}_{0}(\mathbf{x})$, is a deterministic vector, then the material properties in the deformed configuration, $\mathbf{U}(\mathbf{y})$, remains deterministic. In the remainder of this section, it will be assumed that $\left\{\mathbf{U}_{0}(\mathbf{x}), x \in \Omega_{0}\right\}$ is a second-order stochastic field, indexed by $\Omega_{0}$ with values in $\mathbb{R}^{\nu}$. As indicated in Section 2, the Chaos decomposition of $\mathbf{U}_{0}(\mathbf{x})$ can be used to represent it.

\subsubsection{Chaos Decomposition of $\boldsymbol{U}_{0}(\boldsymbol{x})$}

Chaos decompositions provide representations of second-order random variables and processes in terms of Hilbertian bases in $L^{2}$ spaces of random variables. The general infinite-dimensional theory provides for the representation of general second-order variables and processes in terms of Wiener-Hermite polynomials in gaussian random variables $[16,5,14]$. Generalizations, to the case of other polynomials in non-Gaussian variables can be found in [12]. Procedures for identifying the Chaos coefficients in such decompositions, based on experimental data and combining statistical methods with inverse methods for boundary value problems, have also been proposed recently $[4,6,3,1,13,7,10]$ 
Thus starting with experimental data in the natural configuration, a $p$-term Chaos decomposition of the material properties, $\mathbf{U}_{0}(\mathbf{x})$, in this configuration is written as

$$
\mathbf{U}_{0}(\mathbf{x})=\sum_{\alpha=0}^{p} \mathbf{u}_{0 \alpha}(\mathbf{x}) \psi_{\alpha}(\xi),
$$

where $\boldsymbol{\xi}$ is an $\mathbb{R}^{\mu}$-valued random variable with a joint probability measure $P_{\boldsymbol{\xi}}(d \mathbf{s})$ on $\mathbb{R}^{\mu}$. In this equation $\psi_{\alpha}(\boldsymbol{\xi})$ denotes the Hilbertian basis such that

$$
\psi_{0} \equiv 1 \quad\left\langle\psi_{\alpha}(\boldsymbol{\xi}), \psi_{\beta}(\boldsymbol{\xi})\right\rangle=E\left\{\psi_{\alpha}(\boldsymbol{\xi}) \psi_{\beta}(\boldsymbol{\xi})\right\}=\int_{\mathbb{R}^{\mu}} \psi_{\alpha}(\mathbf{s}) \psi_{\beta}(\mathbf{s}) \mathbf{P}_{\boldsymbol{\xi}}(\mathbf{d} \mathbf{s})=\delta_{\alpha \beta},
$$

where $\delta_{\alpha \beta}$ denotes the Kronecker symbol. It is emphasized that as $p, \mu \rightarrow \infty$ the characterization provided by the Chaos decomposition is completely equivalent to that provided by the complete system of joint probability measures. While it is clear that an expression for $\mathbf{u}_{0 \alpha}(\mathbf{x})$ is given by

$$
\mathbf{u}_{0 \alpha}(\mathbf{x})=E\left\{\mathbf{U}_{0}(\mathbf{x}) \psi_{\alpha}(\xi)\right\},
$$

it should be noted that this expression cannot be used for the sake of identifying $\mathbf{u}_{0 \alpha}$ from experimental observations of $\mathbf{U}_{0}(\mathbf{x})$. Alternative identification techniques have been developed for that task.

\subsubsection{Second-Order Moments of Stochastic Field $\boldsymbol{U}_{0}(\boldsymbol{x})$}

From the Chaos representation given by equation (5) it follows that the mean function of $\mathbf{U}_{0}(\mathbf{x}), \mathbf{x} \mapsto \mathbf{m}_{\mathbf{U}_{0}}(\mathbf{x})=E\left\{\mathbf{U}_{0}(\mathbf{x})\right\}$ from $\Omega_{0}$ into $\mathbb{R}^{n}$ is such that

$$
m_{\mathbf{U}_{0}(\mathbf{x})}=\mathbf{u}_{00}(\mathbf{x}) .
$$

The autocorrelation function $\left(\mathbf{x}, \mathbf{x}^{\prime}\right) \mapsto\left[R_{\mathbf{U}_{0}}\left(\mathbf{x}, \mathbf{x}^{\prime}\right)\right]=\mathbf{E}\left\{\mathbf{U}_{\mathbf{0}}(\mathbf{x}) \mathbf{U}_{\mathbf{0}}\left(\mathbf{x}^{\prime}\right)^{\mathbf{T}}\right\}$, from $\Omega_{0} \times \Omega_{0}$ into the set of $n \times n$ real matrices, associated with the $(p, \mu)$ Chaos decomposition, is such that,

$$
\left[R_{\mathbf{U}_{0}}\left(\mathbf{x}, \mathbf{x}^{\prime}\right)\right]=\sum_{\alpha=0}^{p} \mathbf{u}_{0 \alpha}(\mathbf{x}) \mathbf{u}_{0 \alpha}\left(\mathbf{x}^{\prime}\right)^{T}
$$


4.2. Case I: Statistically Invariant Material Properties under Manufacturing Process

In this case, it is assumed that the statistical properties of the material are not modified by the manufacturing process which means that equation (4) holds with $[T(\mathbf{x})]$ a deterministic matrix. The randomness in $\mathbf{U}(\mathbf{y})$, which will be characterized in this section, is solely dependent on the randomness in $\mathbf{U}_{0}(\mathbf{x})$.

\subsubsection{Chaos Decomposition of $\boldsymbol{U}(\boldsymbol{y})$}

From equations (4) and (5) the Chaos decomposition of $\mathbf{U}(\mathbf{y})$ can be deduced in the form,

$$
\mathbf{U}(\mathbf{y})=\sum_{\alpha=0}^{p} \mathbf{u}_{\alpha}(\mathbf{y}) \psi_{\alpha}(\xi)
$$

in which,

$$
\mathbf{u}_{\alpha}(\mathbf{y})=\left([T(\mathbf{x})] \mathbf{u}_{0 \alpha}(\mathbf{x})\right)_{\mathbf{x}=\mathbf{f}^{-1}(\mathbf{y})} .
$$

Thus, the terms in the Chaos representation of $\mathbf{U}(\mathbf{y})$ are transformed individually and identically by $[T(\mathbf{x})]$. It is noted here that this transformation of the statistical content of the material properties becomes significant as the material undergoes finite deformations.

\subsubsection{Second-Order Moments of Stochastic Field $\boldsymbol{U}(\boldsymbol{y})$}

From equation (4), it follows that the mean function of $\mathbf{U}(\mathbf{y}), \mathbf{y} \mapsto \mathbf{m}_{\mathbf{U}}(\mathbf{y})=$ $E\{\mathbf{U}(\mathbf{y})\}$ from $\Omega$ into $\mathbb{R}^{n}$ is such that

$$
\mathbf{m}_{\mathbf{U}}(\mathbf{y})=\left\{[T(\mathbf{x})] \mathbf{m}_{\mathbf{U}_{0}}(\mathbf{x})\right\}_{\mathbf{x}=\mathbf{f}^{-1}(\mathbf{y})} .
$$

From the Chaos representation given by equation (10), $m_{\mathbf{U}}(\mathbf{y})$ can also be expressed as

$$
m_{\mathbf{U}}(\mathbf{y})=\mathbf{u}_{0}(\mathbf{y})
$$

The autocorrelation function $\left(\mathbf{y}, \mathbf{y}^{\prime}\right) \mapsto\left[R_{\mathbf{U}}\left(\mathbf{y}, \mathbf{y}^{\prime}\right)\right]=E\left\{\mathbf{U}(\mathbf{y}) \mathbf{U}\left(\mathbf{y}^{\prime}\right)^{\mathbf{T}}\right\}$, from $\Omega \times$ $\Omega$ into the set of $n \times n$ real matrices can be written as

$$
\left[R_{\mathbf{U}}\left(\mathbf{y}, \mathbf{y}^{\prime}\right)\right]=\left([T(\mathbf{x})]\left[R_{\mathbf{U}_{0}}\left(\mathbf{x}, \mathbf{x}^{\prime}\right)\right]\left[T\left(\mathbf{x}^{\prime}\right)^{T}\right]\right)_{\mathbf{x}=\mathbf{f}^{-1}(\mathbf{y}), \mathbf{x}^{\prime}=\mathbf{f}^{-1}\left(\mathbf{y}^{\prime}\right)} .
$$

Finally, the approximation of $\left[R_{\mathbf{U}}\left(\mathbf{y}, \mathbf{y}^{\prime}\right)\right]$ associated with the $(p, \mu)$ Chaos decomposition of $\mathbf{U}(\mathbf{y})$ given by equation (10) is 


$$
\left[R_{\mathbf{U}}\left(\mathbf{y}, \mathbf{y}^{\prime}\right)\right]=\sum_{\alpha=0}^{p} \mathbf{u}_{\alpha}(\mathbf{y}) \mathbf{u}_{\alpha}\left(\mathbf{y}^{\prime}\right)^{T} .
$$

The identification of coefficients $\mathbf{u}_{\alpha}(\mathbf{y})$ can be achieved either through direct measurements of $\mathbf{U}(\mathbf{y})$ in deformed configuration $\Omega$ or calculated using equation (11). In the first case, the identification process is similar to that described for $\mathbf{u}_{0 \alpha}(\mathbf{x})$ in Section 4.1.1.

\subsubsection{Second-Order Moments for Mean-Square Homogeneous Stochastic Field} $\mathrm{U}_{\mathbf{0}}(\boldsymbol{x})$

Assume that $\mathbf{U}_{0}(\mathbf{x})$ is a second-order mean-square homogeneous stochastic field indexed by $\mathbb{R}^{n}$ with values in $\mathbb{R}^{\nu}$. Consequently, $\mathbf{m}_{\mathbf{U}_{0}}(\mathbf{x})$ is independent of $\mathbf{x}$ and autocorrelation function $\left[R_{\mathbf{U}_{0}}\left(\mathbf{x}, \mathbf{x}^{\prime}\right)\right]$ depends only on $\mathbf{x}-\mathbf{x}^{\prime}$ and is rewritten as $R_{\mathbf{U}_{0}}\left(\mathbf{x}-\mathbf{x}^{\prime}\right)$. From equation (12), it can be seen that the mean function of $\mathbf{U}(\mathbf{y})$ is written as,

$$
\mathbf{m}_{\mathbf{U}}(\mathbf{y})=\left[T\left(\mathbf{f}^{-1}(\mathbf{y})\right)\right] \mathbf{m}_{\mathbf{U}_{0}} .
$$

This equation shows clearly that the mean function of $\mathbf{U}(\mathbf{y})$ will, in general, depend on $\mathbf{y}$. From equation (14), an expression for the autocorrelation function of $\mathbf{U}(\mathbf{y})$ is deduced in the form

$$
\left[R_{\mathbf{U}}\left(\mathbf{y}, \mathbf{y}^{\prime}\right)\right]=\left[T\left(\mathbf{f}^{-1}(\mathbf{y})\right)\right]\left[R_{\mathbf{U}_{0}}\left(\mathbf{f}^{-1}(\mathbf{y})-\mathbf{f}^{-1}\left(\mathbf{y}^{\prime}\right)\right)\right]\left[T\left(\mathbf{f}^{-1}\left(\mathbf{y}^{\prime}\right)\right)\right]^{T}
$$

It is interesting to note that in general, the mean-square homogeneous character of the stochastic field representing the material properties is not preserved under general deformations induced by a manufacturing process. Three cases can be identified in this context.

Rigid Body Motions: . In this case, $\mathbf{x}=\mathbf{f}^{-1}(\mathbf{y})=[\Theta] \mathbf{y}+\mathbf{t}$ where $[\Theta]$ and $\mathbf{t}$ denote an arbitrary invertible real $3 \times 3$ matrix and an arbitrary vector in $\mathbb{R}^{3}$, respectively. It can moreover be shown that in this case, $[T(\mathbf{x})]$ is the identity matrix. Therefore, equations (16) and (17) yield,

$$
\begin{gathered}
\mathbf{m}_{\mathbf{U}}(\mathbf{y})=\mathbf{m}_{\mathbf{U}_{0}}, \\
{\left[R_{\mathbf{U}}\left(\mathbf{y}, \mathbf{y}^{\prime}\right)\right]=\left[R_{\mathbf{U}_{0}}\left([\Theta]\left(\mathbf{y}-\mathbf{y}^{\prime}\right)\right)\right]}
\end{gathered}
$$

These two equations show that $\mathbf{U}(\mathbf{y})$ is a mean-square homogeneous stochastic field on $\mathbb{R}^{n}$. 
Infinitesimal Deformations: This case corresponds to modeling the manufacturing process using linearized elasticity theory. While in this case, $[T(\mathbf{x})]$ remains the identity matrix, the mapping $\mathbf{f}(\mathbf{x})$ is now generally nonlinear. Equations (16) and (17) now yield the following expressions for the mean and autocorrelation functions,

$$
\begin{gathered}
\mathbf{m}_{\mathbf{U}}(\mathbf{y})=\mathbf{m}_{\mathbf{U}_{0}} \\
{\left[R_{\mathbf{U}}\left(\mathbf{y}, \mathbf{y}^{\prime}\right)\right]=\left[R_{\mathbf{U}_{0}}\left(\mathbf{f}^{-1}(\mathbf{y})-\mathbf{f}^{-1}\left(\mathbf{y}^{\prime}\right)\right)\right] .}
\end{gathered}
$$

It is first noted here that $\mathbf{f}^{-1}(\mathbf{y})-\mathbf{f}^{-1}\left(\mathbf{y}^{\prime}\right)$ cannot, in general, be written as $\mathbf{g}\left(\mathbf{y}-\mathbf{y}^{\prime}\right)$ for some $\mathbb{R}^{n}$-valued function $\mathbf{g}$. It is interesting to observe that, in this case, while the mean remains independent of $\mathbf{y}$, the autocorrelation function cannot now be expressed as a function of $\mathbf{y}-\mathbf{y}^{\prime}$, and the process $\mathbf{U}(\mathbf{y})$ is clearly not mean-square homogeneous. It should also be noted here that this situation is typical of many problems that are common both in practice and in research.

Finite Deformation: This case corresponds to modeling the manufacturing process using nonlinear elasticity theory $[2,11,17]$. In this case, $[T(\mathbf{x})]$ is no longer the identity matrix and depends on $\mathbf{x}$. Moreover, the mapping $\mathbf{f}(\mathbf{x})$ is also nonlinear. Equations (16) and (17) now remain in their most general form which are given here again as

$$
\begin{gathered}
\mathbf{m}_{\mathbf{U}}(\mathbf{y})=\left[T\left(\mathbf{f}^{-1}(\mathbf{y})\right)\right] \mathbf{m}_{\mathbf{U}_{0}}, \\
{\left[R_{\mathbf{U}}\left(\mathbf{y}, \mathbf{y}^{\prime}\right)\right]=\left[T\left(\mathbf{f}^{-1}(\mathbf{y})\right)\right]\left[R_{\mathbf{U}_{0}}\left(\mathbf{f}^{-1}(\mathbf{y})-\mathbf{f}^{-1}\left(\mathbf{y}^{\prime}\right)\right)\right]\left[T\left(\mathbf{f}^{-1}\left(\mathbf{y}^{\prime}\right)\right)\right]^{T} .}
\end{gathered}
$$

In this case it is also concluded that the process $\mathbf{U}(\mathbf{y})$ is not, in general, meansquare homogeneous.

\subsection{Case II: Random Evolution of Material Properties under Manufacturing Process}

In this case, it is assumed that the statistical properties of the material are modified by the manufacturing process which means that equation (4) holds with $[T(\mathbf{x})]$ a random matrix. The randomness in $\mathbf{U}(\mathbf{y})$, which will be characterized in this section, now depends on the randomness in both $\mathbf{U}_{0}(\mathbf{x})$ and $[T(\mathbf{x})]$. This situation corresponds to problems where there is uncertainty in the evolution of any given realization of the material properties between configurations $\Omega_{0}$ and $\Omega$. It 
is again reminded that in the present context, the microstructure is assumed to be random. Two nominal cases present themselves, both leading to a matrix $[T(\mathbf{x})]$ which is random. In the first case, the randomness of the microstructure does not change during the manufacturing process, but the manufacturing process itself is random. This case corresponds to matrix-valued random field $\left\{[T(\mathbf{x})], \mathbf{x} \in \Omega_{0}\right\}$ being independent of the $\mathbb{R}^{n}$-valued random field $\left\{\mathbf{U}_{0}(\mathbf{x}), \mathbf{x} \in \Omega\right\}$. In the second case, the randomness of the microstructure changes during the manufacturing process, in addition to the above-mentioned randomness in the process itself. This is the situation, for instance, when the manufacturing process induces new interfaces, stress concentrations, or crack closures/openings in the microstructure. This situation leads to statistical dependence between matrix-valued random field $\left\{[T(\mathbf{x})], \mathbf{x} \in \Omega_{\mathbf{0}}\right\}$ is independent of $\mathbb{R}^{n}$-valued random field $\left\{\mathbf{U}_{0}(\mathbf{x}), \mathbf{x} \in \Omega\right\}$.

Thus two cases will be considered in this section, corresponding to whether the mapping $[T(\mathbf{x})]$ is independent of $\mathbf{U}_{0}(\mathbf{x})$ or not.

\subsubsection{Randomness of Microstructure Unchanged during Manufacturing Process}

In this case, the matrix-valued random field $\left\{[T(\mathbf{x})], \mathbf{x} \in \Omega_{0}\right\}$ is independent of $\mathbb{R}^{n}$-valued random field $\left\{\mathbf{U}_{0}(\mathbf{x}), \mathbf{x} \in \Omega\right\}$. Randomness in $[T(\mathbf{x})]$ necessitates experimental measurements on deformed configuration $\Omega$ in order to identify $\mathbf{U}(\mathbf{y})$ stochastic field. In view of the statistical independence, independent measurements can be justified on $\Omega_{0}$ to identify stochastic field $\mathbf{U}_{0}(\mathbf{x})$ as explained in Section 4.1.

For each $\mathbf{x}$ fixed in $\Omega_{0}$ let $\mathbf{U}_{0}\left(\mathbf{x}, \kappa_{j}\right), j=1, \ldots, J$ be $J$ independent realizations of $\mathbf{U}_{0}(\mathbf{x})$. These correspond to different realizations of the random initial microstructure. Each realization $\mathbf{U}_{0}\left(\mathbf{x}, \kappa_{j}\right)$ is transformed, during the same realization $\theta_{j}$ of the manufacturing process, into $\mathbf{U}\left(\mathbf{y}, \theta_{j}, \kappa_{j}\right)$. From equation (4), it is deduced that

$$
\mathbf{U}\left(\mathbf{y}, \theta_{j}, \kappa_{j}\right)=\left[T\left(\mathbf{x}, \theta_{j}\right)\right] \mathbf{U}_{0}\left(\mathbf{x}, \kappa_{j}\right), \quad \mathbf{y}=\mathbf{f}(\mathbf{x})
$$

in which $\left[T\left(\mathbf{x}, \theta_{j}\right)\right]$ is a realization of random matrix $[T(\mathbf{x})]$. It is clear from the above equation that the statistical identification of random matrix $[T(\mathbf{x})]$ is an experimentally unrealistic problem. At each point $\mathbf{x}$, the same realization of the manufacturing process must be applied to different realizations of the initial microstructure. This is clearly physically unrealizable, in the context of a random manufacturing process. Assuming that such a hurdle could be overcome, the estimation of a single almost surely invertible realization random matrix $[T(\mathbf{x})]$ still requires at $\nu^{2}$ experimental observations. Thus for an anisotropic material for which $\nu=21$, we need 441 experimental tests. 
This analysis strongly suggests the need, in such problems, for an a priori stochastic representation for the manufacturing process.

\subsubsection{Randomness of Microstructure Evolving during Manufacturing Process}

This last case to be considered addresses situations where the evolution of the material parameters during the manufacturing process is statistically dependent on the initial configuration of the microstructure. In this case, equation (4), can be written down for a given experimental realization if the form,

$$
\mathbf{U}\left(\mathbf{y}, \theta_{j}, \kappa_{j}\right)=\left[T\left(\mathbf{x}, \theta_{j}, \kappa_{j}\right)\right] \mathbf{U}_{0}\left(\mathbf{x}, \kappa_{j}\right), \quad \mathbf{y}=\mathbf{f}(\mathbf{x}),
$$

where now each realization of random matrix $[T(\mathbf{x})]$, in addition of being dependent on $\theta_{j}$, also depends on $\kappa_{j}$. It is therefore obvious that the experimental identification of models to be used in support of such analyses is quite unrealistic.

\section{Conclusion}

A detailed analysis has been presented of the analysis of stochastic processes used in representing material properties. It has been found that the mathematical structure of these processes is highly dependent on the deformation processes that these materials undergo. Two particular cases are worth noting. The first case deals with problems where the statistical properties of the material microstructure do not change during the manufacturing process. For this class of problems, a method to characterize the probabilistic structure of these materials in their manufactured configuration, which is the configuration used for numerical predictions, has been proposed. It should be noted that the probabilistic structure of the material properties in this configuration are not very intuitive and disagree with current practice. The second class of problems deals with situations where the statistical properties of the material microstructure change during the manufacturing process. A treatment of these problems does not seem to be accessible to a direct experimental identification. This suggests the need for further investigations for modeling the effects of the random microstructure at the macroscale. It is imperative in developing such models to be mindful of the experimental identification process required by such models. Recent developments in probabilistic models pave the way for the modeling of random microstructure with minimal parametrization. 


\section{References}

[1] Arnst, M., Ghanem, R., and Soize, C., Identification of Bayesian posteriors for coefficients of chaos expansions. Journal of Computational Physics, 229(9), pp. 3134-3154, 2010.

[2] Ciarlet, P. G., Mathematical Elasticity, Vol. 1, North-Holland, Amsterdam, 1988.

[3] Das, S., Ghanem, R., and Spall, J. C., Asymptotic sampling distribution for polynomial chaos representation from data: a maximum entropy and fisher information approach. SIAM Journal of Scientific Computing, 30(5), pp. 2207-2234, 2008.

[4] Desceliers, C., Ghanem, R., and Soize, C., Maximum likelihood estimation of stochastic chaos representations from experimental data. International Journal for $\mathrm{Nu}$ merical Methods in Engineering, 66(6), pp. 978-1001, 2006.

[5] Ghanem, R., and Spanos, P. D., Stochastic Finite Elements: A spectral Approach (revised edition). Dover Publications: New York, 2003. First edition, Springer-Verlag, New York, 1991.

[6] Ghanem, R., and Doostan, R., Characterization of stochastic system parameters from experimental data: A bayesian inference approach. Journal of Computational Physics, 217(1), pp. 63-81, 2006.

[7] Guilleminot, J., and Soize, C., Stochastic model and generator for random fields with symmetry properties: application to the mesoscopic modeling of elastic random media. Multiscale Modeling and Simulation (A SIAM Interdisciplinary Journal), 11(3), pp. 840-870, 2013.

[8] Kunin, I. A., Elastic Media with Microstructure, Vol 1: One-Dimensional Models, Springer-Verlag, Heidelberg, 1982.

[9] Kunin, I. A., Elastic Media with Microstructure, Vol 2: Three-Dimensional Models, Springer-Verlag, Heidelberg, 1983.

[10] Nouy, A., and Soize, C., Random fields representations for stochastic elliptic boundary value problems and statistical inverse problems, European Journal of Applied Mathematics, 25(3), pp. 339-373, 2014.

[11] Ogden, R. W., Non-linear Elastic Deformations, Ellis Horwood, New York, 1984.

[12] Soize, C., and Ghanem, R., Physical Systems with Random Uncertainties: Chaos Representations with Arbitrary Probability Measure. SIAM Journal on Scentific Computing, 26(2), pp. 395-410, 2004. 
[13] Soize, C., Identification of high-dimension polynomial chaos expansions with random coefficients for non-Gaussian tensor-valued random fields using partial and limited experimental data. Computer Methods in Applied Mechanics and Engineering, 199(33-36), pp. 2150-2164, 2010.

[14] Tipireddy, R., and Ghanem, R., Basis adaptation in homogeneous chaos spaces. Journal of Computational Physics, 259, pp. 304-317, 2014.

[15] Torquato, S., Random Heterogeneous Materials : Microstructure and Macroscopic Properties, Springer, Berlin, 2002.

[16] Wiener, N., The Homogeneous Chaos, American Journal of Mathematics, 60(4), pp. 897-936, 1938.

[17] Zienkiewicz, O. C., and Taylor, R. L., The Finite Element Method. ButterworthHeinemann, Oxford, 2000. 